\title{
LISTADO PRELIMINAR DE AUCHENORRHYNCHA (INSECTA: HEMIPTERA) DE LA RESERVA ECOLÓGICA DEL PEDREGAL DE SAN ÁNGEL, DISTRITO FEDERAL, MÉXICO
}

\author{
RICARDO MARIÑO-PÉREZ, ${ }^{1}$ ILIANA PACHECO-RUEDA, ${ }^{2}$ CHRISTOPHER \\ DIETRICH $^{3}$ \\ ${ }^{1}$ Universidad Nacional Autónoma de México, Facultad de Ciencias, 04510 México D.F. <pselliopus@ \\ yahoo.com.mx> \\ ${ }^{2}$ Colegio de Posgraduados, Instituto de Fitosanidad, Montecillo, 56230 Texcoco, Edo. de México, \\ México.<atta_mexicana@yahoo.com.mx> \\ 3Illinois Natural History Survey, 1816 Oak street, IL 61820 < dietrich@inhs.illinois.edu>
}

Mariño-Pérez, R., I. Pacheco-Rueda \& C. Dietrich. 2012. Listado preliminar de Auchenorrhyncha (Insecta: Hemiptera) de la Reserva Ecológica del Pedregal de San Ángel, Distrito Federal, México. Acta Zoológica Mexicana (n. s.), 28(2): 280-286.

RESUMEN. Se aportan datos sobre la fauna de Auchenorrhyncha de la Reserva Ecológica del Pedregal de San Ángel, Distrito Federal, para conocer su diversidad. Se encontraron 33 especies pertenecientes a 8 familias, 14 subfamilias y 28 géneros. Se trata del primer listado ilustrado para esta área, que proporciona su ubicación y tipo de vegetación, la cual es única, bajo protección especial de la UNAM y en proceso de ingresar al Sistema Nacional de Áreas Naturales Protegidas.

Palabras clave: Auchenorrhyncha, Pedregal de San Ángel, área protegida, riqueza de especies.

Mariño-Pérez, R., I. Pacheco-Rueda \& C. Dietrich. 2012. Preliminary checklist of Auchenorrhyncha (Insecta: Hemiptera) from Reserva Ecológica del Pedregal de San Ángel, Distrito Federal, Mexico. Acta Zoológica Mexicana (n. s.), 28(2): 280-286.

ABSTRACT. We provide data on the fauna of Auchenorrhyncha from the Reserva Ecológica del Pedregal de San Ángel, Distrito Federal, to know its diversity. Thirty three species belonging to 8 families, 14 subfamilies and 28 genera were found. This is the first illustrated checklist for this area that, due to its location, origin and vegetation, is unique, protected by the UNAM and in process of being incorporated to the National System of Protected Natural Areas.

Keywords: Auchenorrhyncha, Pedregal de San Ángel, protected area, species richness.

\section{INTRODUCCIÓN}

La Reserva Ecológica del Pedregal de San Ángel forma parte de la Universidad Nacional Autónoma de México (UNAM), comprende una superficie de 237 hectáreas

Recibido: 23/11/2010; aceptado: 21/02/2012. 
de un ecosistema endémico formado por el derrame de lava del volcán Xitle, hace aproximadamente 2000 años. La zona, por su particular y único paisaje, llamó la atención de muchos viajeros y exploradores célebres, entre ellos Alexander von Humboldt y Andrés Manuel del Río, quienes la visitaron a principios del siglo XIX (Cano-Santana 1994). En esta zona predomina una flora de matorral xerófito, a la cual Rzedowski (1954) denominó Senecionetum praecocis, la planta que actualmente da nombre a la formación vegetal es Pittocaulon praecox (Cav.) Rob \& Bret [Asteraceae] (ex Senecio praecox) (Olson 2005). El tipo de clima que presenta la zona de la reserva es templado subhúmedo, con una precipitación anual de 700 a $900 \mathrm{~mm}$, y altitudes entre 2,200 y 2,277 msnm (REPSA 2006). Estas condiciones han originado una diversidad específica de insectos muy alta (Lot \& Cano-Santana 2009).

El suborden de hemípteros Auchenorrhyncha comprende a los insectos chupadores de savia de las superfamilias Cercopoidea (salivazos; Cercopidae, Aphrophoridae), Cicadoidea (cigarras; Cicadidae), Membracoidea (chicharritas y periquitos; Cicadellidae, Membracidae) y Fulgoroidea (Cixiidae, Derbidae, Flatidae, Issidae). Estos grupos en conjunto incluyen más de 40,000 especies descritas a nivel mundial, morfológicamente difieren de otros hemípteros porque el rostrum se encuentra en la superficie posteroventral de la cabeza (Dietrich 2003). Se trata de insectos abundantes, distribuidos mundialmente en casi todos los hábitats terrestres, y son particularmente diversos y con gran riqueza de especies en los trópicos. Algunos son plagas de plantas, afectándoles ya sea por alimentarse de ellas, depositar sus huevecillos en ellas o por transmitirles patógenos como virus (Dietrich 2003). El objetivo de este estudio fue conocer la fauna de Auchenorrhyncha de la Reserva Ecológica del Pedregal de San Ángel.

\section{MATERIALES Y MÉTODOS}

La recolección del material biológico se realizó de febrero a diciembre de 2006 en la Reserva Ecológica del Pedregal de San Ángel. Se recolectaron exclusivamente adultos, los cuales se atraparon con un aspirador entomológico y con la ayuda de una red de golpeo. Posteriormente se fijaron en alcohol al 70\%. Se montaron en alfiler entomológico para ser identificados con las claves de Crowder (1952), DeLong y Hamilton (1974), Dietrich y Deitz (1991), Doering y Shepherd (1946), Hamilton (1977, 1998), Hepner (1947), Nast (1949), Nielson (1982) y Young (1977). El material que se identificó está depositado en la Colección Nacional de Insectos (CNIN) del Instituto de Biología de la Universidad Nacional Autónoma de México, en México, D. F., y en la Colección Entomológica del Illinois Natural History Survey (INHS), en Urbana, Illinois, E. U. 


\section{RESULTADOS}

En las seis recolectas realizadas en la Reserva Ecológica del Pedregal de San Ángel, se capturaron 197 ejemplares pertenecientes a ocho familias, 14 subfamilias, 28 géneros y 33 especies (Cuadro 1). La razón por la que algunas especies únicamente se determinaron hasta género es por haber colectado sólo hembras. El siguiente número de especies fue registrada por muestreo: febrero (4), marzo (3), mayo (7), julio (16); agosto (8), y diciembre (9). Treinta especies fueron recolectadas solo en uno o dos muestreos, únicamente Chlorogonalia losoplanensis y Balclutha impicta fueron recolectadas en tres muestreos y Graphocephala appropinquans se encontró en cuatro muestreos. El esfuerzo de colecta por muestreo siempre fue aproximadamente de ocho horas (dos personas colectando durante cuatro horas).

La familia Cicadellidae fue la más abundante con 23 especies, seguida por Cercopidae, Cixiidae y Membracidae con dos especies cada una y el resto de las familias (Aphrophoridae, Derbidae, Flatidae e Issidae) con una especie, cada una. En la Lámina I se ilustran fotográficamente diez especies, que representan a las principales familias.

\section{DISCUSIÓN}

La gran mayoría de las especies tratadas en este estudio tienen una amplia distribución, no obstante juegan un papel muy importante en la cadena trófica de este ecosistema endémico, siendo uno de los principales componentes de la biomasa animal. Respecto a registros previos, Cano-Santana y Oyama (1994) reportan al membrácido Aconophora pallescens y al cicadélido Graphocephala sp. asociados a la planta Wigandia urens (Hydrophyllaceae). Respecto a la primera especie, se le puede considerar como presente, debido a su amplia distribución. Para el caso de la segunda, nosotros reportamos dos especies de Graphocephala (G. appropinquans y G. flavovittata), y dada la presencia en cuatro muestreos de G. appropinquans, muy probablemente se trate de esta especie.

La Reserva Ecológica del Pedregal de San Ángel, localizada en el Campus Universitario de la UNAM, es uno de los últimos lugares que alberga la biodiversidad silvestre del matorral de Pittocaulon praecox (palo loco), la cual coexiste con uno de los sistemas urbanos más complejos y contaminados del mundo. A pesar de que otras áreas de la Ciudad de México presentan matorral xerófito (Bosque de Tlalpan y el Parque Ecológico de Cuicuilco), no están adecuadamente conservadas, por tratarse de parques abiertos al público, por lo que la conservación de este tipo de matorral recae en el área de la reserva. Algunos grupos de insectos han sido estudiados en esta reserva (Lot y Cano-Santana 2009); sin embargo, este es el primer trabajo realizado en este lugar para el grupo, que incluye un registro fotográfico con la diversidad de Auchenorrhyncha en un área relativamente pequeña en comparación con otros lugares de importancia biológica. Se trata de un listado preliminar, ya que no se realizaron 
Cuadro 1. Listado preliminar de Auchenorryncha de la Reserva Ecológica del Pedregal de San Ángel.

\begin{tabular}{|c|c|c|}
\hline Familia & Subfamilia & Especie \\
\hline \multirow[t]{6}{*}{ Cicadellidae } & Cicadellinae & $\begin{array}{l}\text { Amphigonalia aridella (DeLong, 1948) } \\
\text { *Apogonalia mediolineata (Fowler, 1899) } \\
\text { *Chlorogonalia losoplanensis (Schroeder, 1959) } \\
\text { Draeculacephala sp. } \\
\text { Graphocephala appropinquans (Fowler, 1899) } \\
\text { Graphocephala flavovittata Metcalf, } 1955 \\
\text { Homalodisca elongata Ball, } 1936 \\
\text { Sibovia sp. }\end{array}$ \\
\hline & Coelidiinae & *Omanolidia bistyla Nielson, 1982 \\
\hline & Deltocephalinae & $\begin{array}{l}\text { Amblysellus necopinus DeLong \& Hamilton, } 1974 \\
\text { Amblysellus punctatus (Osborn \& Ball, 1898) } \\
\text { Balclutha impicta (Van Duzee, 1892) } \\
\text { Exitianus brevis DeLong \& Hershberger, } 1947 \\
\text { *Exitianus picatus Gibson, } 1919 \\
\text { Polyamia reticulata (Linnavuori, 1959) } \\
\text { Stirellus bicolor (Van Duzee, 1892) } \\
\text { Scaphytopius pennatus Hepner, 1946 } \\
\text { Scaphytopius sp. } \\
\text { *Texananus sp. cercana a sextus Hepner, } 1946\end{array}$ \\
\hline & Iassinae & Penestragania sp. \\
\hline & Megophthalminae & *Ceratagallia sordida (Oman, 1933) \\
\hline & Typhlocybinae & $\begin{array}{l}\text { Empoasca sp. } 1 \\
\text { Empoasca sp. }\end{array}$ \\
\hline Aphrophoridae & Aphrophorinae & Lepyronia sp. \\
\hline Cercopidae & Tomaspidinae & $\begin{array}{l}\text { *Ocoaxo punctus (Nast, 1949) } \\
\text { *Prosapia ignifera Hamilton, } 1977\end{array}$ \\
\hline Cixiidae & Cixiinae & $\begin{array}{l}\text { Melanoliarus sp. } \\
\text { Cixius sp. }\end{array}$ \\
\hline Derbidae & Derbinae & Cedusa sp. \\
\hline Flatidae & Flatinae & *Mistharnophantia curta Doering \& Shepard, 1946 \\
\hline Issidae & Issinae & Hysteropterum sp. \\
\hline \multirow[t]{2}{*}{ Membracidae } & Smiliinae & Polyglypta sp. \\
\hline & Membracinae & *Aconophora laminata Fairmaire, 1846 \\
\hline
\end{tabular}

*Especie fotografiada.

muestreos nocturnos con trampa de luz ni otros métodos diurnos como succión con aspiradora de motor; solo fue utilizada la red de golpeo y la colecta manual, y es un primer paso para conocer la fauna de auquenorrincos del área, y sirve de base para que en futuros estudios y usando todos los métodos de colecta se obtenga el listado 


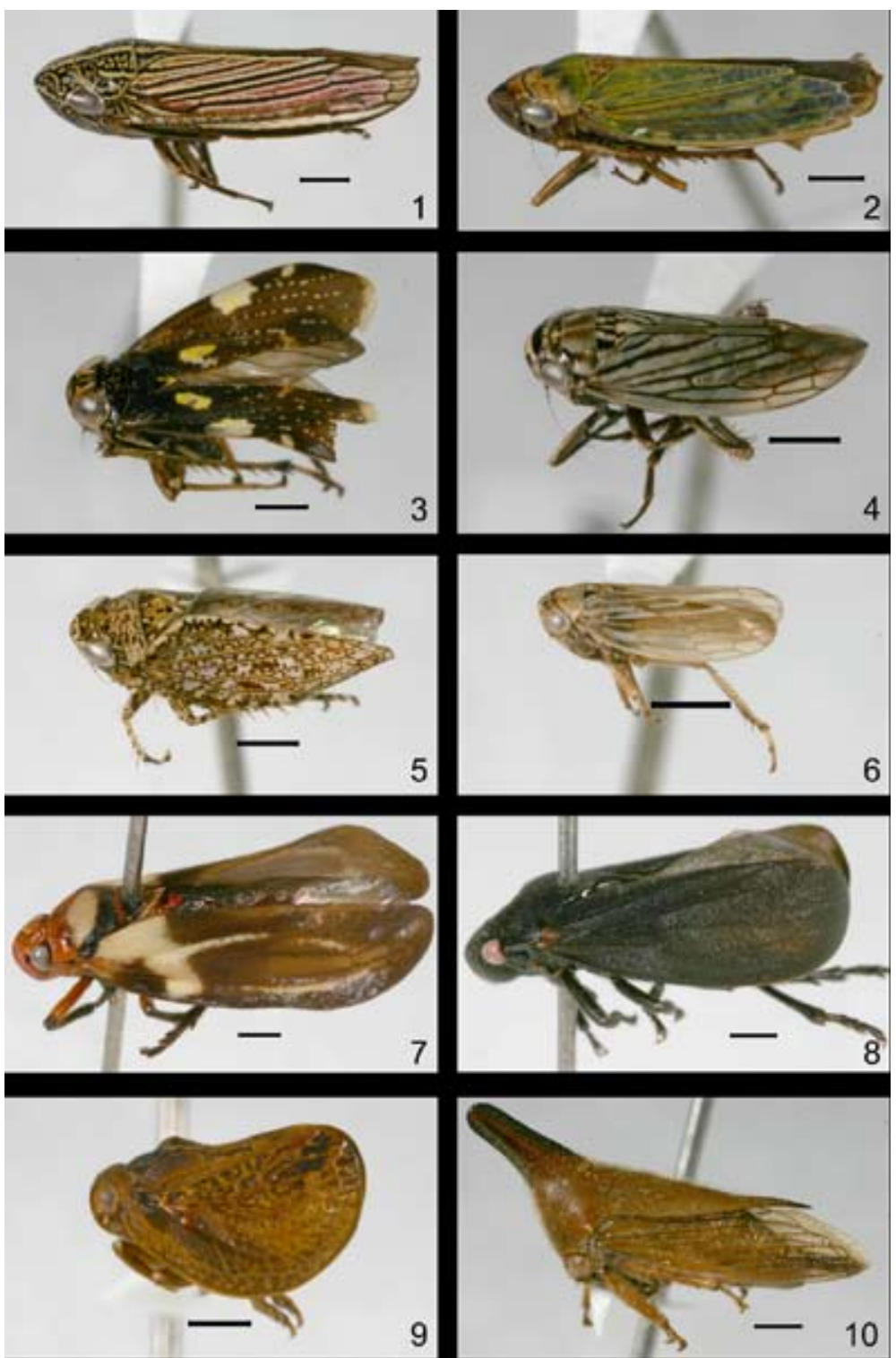

Lámina I. Diversidad de Auchenorrhyncha en el Pedregal de San Ángel. 1. Apogonalia mediolineata

(Cicadellidae; Cicadellinae). 2. Chlorogonalia losoplanensis (Cicadellidae; Cicadellinae). 3.

Omanolidia bistyla (Cicadellidae; Coelidiinae). 4. Exitianus picatus (Cicadellidae; Deltocephalinae). 5. Texananus sp. cercana a sextus (Cicadellidae; Deltocephalinae). 6. Ceratagallia sordida (Cicadellidae;

Megophthalminae). 7. Ocoaxo punctus (Cercopidae; Tomaspidinae). 8. Prosapia ignifera

(Cercopidae; Tomaspidinae). 9. Mistharnophantia curta (Flatidae; Flatinae). 10. Aconophora laminata (Membracidae; Membracinae). Barra de referencia $=1 \mathrm{~mm}$. 
completo, fundamental para conocer qué especies existen, para entender y conservar adecuadamente la reserva, que aunque pequeña, es de gran importancia por encontrarse dentro de la Ciudad de México y por la formación de rocas basálticas en la reserva, que posee un alto valor biológico, ecológico y geomorfológico, además de que recarga los acuíferos, mantiene la humedad y la calidad del aire, y contribuye a amortiguar los cambios de temperatura en el microclima.

Algunas especies únicamente se determinaron a nivel de género, por contarse sólo con hembras, por lo que colectas subsecuentes son importantes para encontrar a los machos y verificar su identidad además de obtener datos sobre sus hospederos y la eventual descripción de especies nuevas.

Agradecimientos. Al Consejo Nacional de Ciencia y Tecnología (CONACYT) por la beca otorgada al primer autor, que le permitió realizar una estancia en el Illinois Natural History Survey para concluir este artículo. Al Dr. Zenón Cano Santana, de la Facultad de Ciencias, Universidad Nacional Autónoma de México, por las facilidades otorgadas para el trabajo en la reserva, y por habernos inculcado un gran amor hacia este lugar. A tres revisores, cuyas sugerencias mejoraron la calidad de este manuscrito.

\section{LITERATURA CITADA}

Cano-Santana, Z. 1994. Flujo de energía a través de Sphenarium purpurascens (Orthoptera: Acrididae) y productividad primaria neta aérea en una comunidad xerófita. Tesis de Doctorado. Universidad Nacional Autónoma de México, México D.F. 235 pp.

Cano-Santana, Z. \& K. Oyama. 1994. Wigandia urens (Hydrophyllaceae): Un mosaico de recursos para sus insectos herbívoros. Acta Botánica Mexicana, 28: 29-39.

Crowder, H. W. 1952. A revision of some Phlepsius like genera of the tribe Deltocephalini (Homoptera, Cicadellidae) in America north of Mexico. University of Kansas Science Bulletin, 35: 309-541.

DeLong, D. M., \& K. G. A. Hamilton. 1974. The genus Amblysellus (Homoptera: Cicadellidae): a key to the known species, with descriptions of eight new species. Canadian Entomologist, 106: 841-849.

Dietrich, C. H. 2003. Auchenorrhyncha (Cicadas, Spittlebugs, Leafhoppers, Treehoppers and Planthoppers). pp. 66-74. In: V. H. Resh \& R. Cardé (Eds.). Encyclopedia of Insects. Elsevier Science, San Diego, CA. EUA.

Dietrich, C. H. \& L. L. Deitz. 1991. Revision of the Neotropical treehopper tribe Aconophorini (Homoptera: Membracidae). North Carolina Agricultural Research Service Technical Bulletin, 293: 1-134.

Doering, K. C. \& C. J. Shepherd. 1946. Some new species of the genus Mistharnophantia, Kirkaldy (Subfamily Flatinae, Family Fulgoridae, Homoptera). Journal of the Kansas Entomological Society, 19: 111-127.

Hamilton, K. G. A. 1977. Review of the world species of Prosapia Fennah (Rhynchota: Homoptera: Cercopidae). Canadian Entomologist, 109: 621-630.

Hamilton, K. G. A. 1998. The species of the North American leafhoppers Ceratagallia Kirkaldy and Aceratagallia Kirkaldy (Rhynchota: Homoptera: Cicadellidae). Canadian Entomologist, 130: 427490.

Hepner, L. W. 1947. A revision of the tribe Scaphytopini (Homoptera, Cicadellidae). North America. University of Kansas Science Bulletin, 31: 413-541. 
Lot, A. \& Z. Cano-Santana. 2009. Biodiversidad del ecosistema del Pedregal de San Ángel. UNAM, Coordinación de la Investigación Científica, 538 pp.

Nast, J. 1949. A revision of the genus Sphenorhina Am. et Serv. (Homoptera, Cercopidae). Polskie Pismo Entomologiczne, 19: 114-148.

Nielson, M. W. 1982. A revision of the subfamily Coelidiinae (Homoptera: Cicadellidae) IV. Tribe Coelidiini. Pacific Institutional Monographs, 38: 1-318.

Olson, M. E. 2005. Wood, bark, and pith anatomy in Pittocaulon ( Senecio, Asteraceae): Water storage and systematics. Journal of the Torrey Botanical Society, 132: 173-186.

Rzedowski, J. 1954. Vegetación del Pedregal de San Ángel (D.F, México). Anales de la Escuela Nacional de Ciencias Biológicas, Instituto Politécnico Nacional, 8: 59-129.

REPSA. 2006. Reserva Ecológica del Pedregal de San Ángel. Consultado en línea el 2 de diciembre de 2006. http://www.repsa.unam.mx/

Young, D. A. 1977. Taxonomic study of the Cicadellinae (Homoptera: Cicadellidae). Part II. New World Cicadellini and the genus Cicadella. North Carolina Agricultural and Experimental State Technical Bulletin, 239: 1-1135. 